
Key Insights from Structural Studies of High-Temperature Superconductors: Is There
a Path to Higher Tc?

\author{
J.D. Jorgensen \\ Materials Science Division and Science and Technology Center for Superconductivity, Argonne \\ National Laboratory, Argonne, IL 60439
}

\begin{abstract}
The submitted manuscript has been created by the University of Chicago as Operator of Argonne National Laboratory ("Argonne") under Contract No. W-31-109-ENG-38 with the U.S. Department of Energy. The U.S. Government retains for itself, and others acting on its behalf, a paid-up, nonexclusive, irrevocable worldwide license in said article to reproduce, prepare derivative works, distribute copies to the public, and perform publicly and display publicly, by or on behalf of the Government.
\end{abstract}

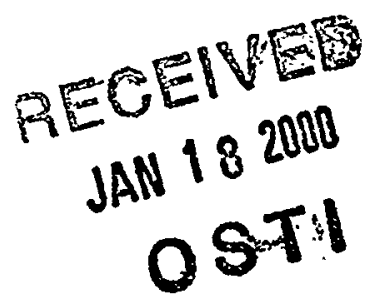

October 1999

Invited Paper submitted to the 12rh International Symposium on Superconductivity (ISS'99), Morioka, Japan, 17-19, 1999

\title{
Distribution:
}

\section{1-2. PRS}

3. J. M. Gibson

4. G. W. Crabtree

5-6 J. D. Jorgensen (2)

7 Janice Coble

8-11. Authors

This work is supported by the US Department of Energy, Office of Science - Materials Sciences, under Contract No. W-31-109-ENG-38, and the NSF Office of Science and Technology Centers, Grant No. DMR 91-20000. 


\section{DISCLAIMER}

This report was prepared as an account of work sponsored by an agency of the United States Government. Neither the United States Government nor any agency thereof, nor any of their employees, make any warranty, express or implied, or assumes any legal liability or responsibility for the accuracy, completeness, or usefulness of any information, apparatus, product, or process disclosed, or represents that its use would not infringe privately owned rights. Reference herein to any specific commercial product, process, or service by trade name, trademark, manufacturer, or otherwise does not necessarily constitute or imply its endorsement, recommendation, or favoring by the United States Government or any agency thereof. The views and opinions of authors expressed herein do not necessarily state or reflect those of the United States Government or any agency thereof. 


\section{DISCLAIMER}

\section{Portions of this document may be illegible in electronic image products. Images are produced from the best available original document:}




\title{
Key Insights from Structural Studies of High-Temperature Superconductors: Is There a Path to Higher $\mathbf{T}_{\mathbf{c}}$ ?
}

\author{
James D. Jorgensen
}

Materials Science Division and Science and Technology Center for Superconductivity, Argonne National Laboratory, Argonne, IL 60439 USA

\begin{abstract}
Structural studies have allowed the development of a model for the "ideal" high-temperature superconductor. For a given compound, the maximum $T_{c}$ is traditionally achieved by using a chemical variable to adjust the carrier concentration to the optimum value. When comparing different compounds at their optimum doping, the highest $\mathrm{T}_{c}$ is observed for compounds with flat $\mathrm{CuO}_{2}$ planes. $\mathrm{T}_{c}$ can also be enhanced if the charge reservoir region, or blocking layer, is metallic. In general, these three criteria cannot simultaneously be met by adjusting a single chemical/structural variable. Additionally, recent work on $\mathrm{HgBa}_{2} \mathrm{CuO}_{4+\mathrm{x}}$ and 123 compounds as a function of doping suggest that electronically-driven structural distortions may hinder attempts to produce higher $\mathrm{T}_{c}$ 's by chemical substitutions. In spite of these challenges, the ideal high- $T_{\mathcal{C}}$ compound has not yet been discovered and the search should continue.
\end{abstract}

Keywords: Crystal structure, Maximum $\mathrm{T}_{\mathrm{c}}$, Doping, Electronic Structure, Electronically-driven structural instability

\section{INTRODUCTION}

Although over fifty distinct copper-oxide superconductors have been discovered since 1986 [1], the superconducting transition temperature, $\mathrm{T}_{\mathrm{C}}$, (at ambient pressure) has not been raised above $135 \mathrm{~K}$, which was achieved in the three-layer $\mathrm{HgBa}_{2} \mathrm{Ca}_{2} \mathrm{Cu}_{3} \mathrm{O}_{8+\mathrm{x}}$ compound in 1993.[2] Nevertheless, the wide variety of structural features manifest by these compounds has led to a consensus concerning what chemical/structural features give rise to the highest $T_{c}$. In this paper, $I$ review these conclusions and discuss the challenge of finding new compounds with higher $T_{c}$ 's.

\section{OPTIMUM DOPING}

The first critical insight from structural studies was that chemical modification of the charge reservoir layer (also called the blocking. layer) could be used to create carriers in the metallic $\mathrm{CuO}_{2}$ conduction layer which is responsible for the superconductivity. Perhaps the most convincing demonstration of this principle was that the bond valence sum for the $\mathrm{Cu}$ atoms in the $\mathrm{CuO}_{2}$ planes in $\mathrm{YBa}_{2} \mathrm{Cu}_{3} \mathrm{O}_{6+\mathrm{x}}$ scaled with the oxygen content in the $\mathrm{CuO}_{x}$ chains in the charge reservoir layer.[3] This bond valence sum is an estimate of the charge at the plane $\mathrm{Cu}$ site calculated from the lengths of the $\mathrm{Cu}-\mathrm{O}$ bonds around this site. This concept of creating carriers in the $\mathrm{CuO}_{2}$ planes through chemical modification of the charge reservoir layer came to be known as the charge transfer model.

Consistent with this hypothesis, it was found that a variety of defects could be used in the charge reservoir layer to control the concentration of charge carriers. For example, in the 
insulating $\mathrm{La}_{2} \mathrm{CuO}_{4}$ compound, superconductivity can be created by substitution of a $2+$ cation (e.g., $\mathrm{Ba}, \mathrm{Sr}$, or $\mathrm{Ca}$ ) on the $\mathrm{La}^{3+}$ site or by insertion of interstitial oxygen defects in the $\mathrm{La}_{2} \mathrm{O}_{2}$ charge reservoir layer.[4] However, the relationship between the charge transfer and the defect chemistry can be rather complex, such as the case where defect ordering in the chain region of $\mathrm{YBa}_{2} \mathrm{Cu}_{3} \mathrm{O}_{6+x}$, at constant oxygen content, dramatically affects the charge transfer and the $T_{C}$. [5]

\section{DEFECTS IN THE $\mathrm{CuO}_{2}$ LAYERS}

Whereas defects in the charge reservoir layer may be required to achieve the desired carrier concentration, defects in or near the $\mathrm{CuO}_{2}$ layers are clearly detrimental to superconductivity. This is especially true for the substitution of metal atoms on the plane $\mathrm{Cu}$ site.[6] Small concentrations of such defects destroy superconductivity. $(\mathrm{La}, \mathrm{Sr}, \mathrm{Ca})_{3} \mathrm{Cu}_{2} \mathrm{O}_{6+x}$ is a particularly interesting system for the study of defects near the $\mathrm{CuO}_{2}$ layers. This is the two-layer compound in the series beginning with $\mathrm{La}_{2} \mathrm{CuO}_{4}$. When first discovered, $\mathrm{La}_{2} \mathrm{SrCu}_{2} \mathrm{O}_{6}$ was found to be metallic, but, mysteriously, not superconducting. It was subsequently shown that substitution of a small $\mathrm{Ca}$ cation at the metal site between the two $\mathrm{CuO}_{2}$ planes (analogous to the $\mathrm{Y}$ site in $\mathrm{YBa}_{2} \mathrm{Cu}_{3} \mathrm{O}_{7}$ ) produced superconductivity by reducing the dimensions of the structure in this region and eliminating the formation of an interstitial oxygen defect within the double $\mathrm{CuO}_{2}$ layer.[7] The formation of defects in or near the $\mathrm{CuO}_{2}$ layers may explain why some metallic layered copper-oxide compounds do not exhibit superconductivity.

\section{OPTIMUM STRUCTURE FOR HIGH $\mathbf{T}_{c}$}

The observation of a wide range of maximum $T_{c}$ 's in the various copper-oxide superconductors, after carrier concentration is optimized and detrimental defects are eliminated in or near the $\mathrm{CuO}_{2}$ layers, argues that there is an optimum structure for achieving the highest $T_{c}$. Conclusions about the features of this optimum structure come from comparing different compounds. The highest $\mathrm{T}_{c}$ 's are observed for compounds with flat $\mathrm{CuO}_{2}$ planes. The $\mathrm{HgBa}_{2} \mathrm{Ca}_{n-1} \mathrm{Cu}_{n} \mathrm{O}_{2 n+2+x}$ compounds satisfy this criteria. The $\mathrm{n}=1,2$, and 3 members of this series exhibit the highest $T_{c}$ 's reported for any one-, two-, or three-layer compounds (Table 1). The important feature of the structures of these three compounds is that the strong bonding of the apical oxygen atom to $\mathrm{Hg}$ results in a weak, and unusually long, bond of this oxygen atom to the plane $\mathrm{Cu}$ atom. This long apical $\mathrm{Cu}-\mathrm{O}$ bond reduces the repulsion between the apical oxygen atom and the oxygen atoms in the $\mathrm{CuO}_{2}$ plane, allowing flat $\mathrm{CuO}_{2}$ planes.

Table 1. Buckling angles of the $\mathrm{CuO}_{2}$ planes $(\mathrm{Cu}-\mathrm{O}-\mathrm{Cu})$ and copper-oxygen apical bond lengths ( $\mathrm{Cu}-\mathrm{O})$ for $\mathrm{HgBa}_{2} \mathrm{Ca}_{n-1} \mathrm{Cu}_{n} \mathrm{O}_{2 n+2+x}$ compounds. Numbers in parentheses are standard deviations of the last significant digit. (from Refs. 8,9,10)

\begin{tabular}{cccc}
\hline No. of layers, $\mathrm{n}$ & $\mathrm{T}_{\mathrm{c}}(\mathrm{K})$ & $\mathrm{Cu}-\mathrm{O}-\mathrm{Cu}\left(^{\circ}\right)$ & $\mathrm{Cu}-\mathrm{O}(\mathrm{A})$ \\
\hline 1 & 95 & 180 & $2.780(1)$ \\
2 & 126 & $179.4(2)$ & $2.775(3)$ \\
3 & 135 & $178.4(4)$ & $2.741(6)$ \\
\hline
\end{tabular}

The importance of the buckling angle of the $\mathrm{CuO}_{2}$ planes can be seen by comparing the $\mathrm{T}_{c}$ 's and buckling angles for several compounds, as shown in Fig. 1.[11] Only compounds with two $\mathrm{CuO}_{2}$ layers are shown in this figure because the most accurate structural data are available for these compounds. The same behavior is observed for three- 
layer compounds, but with fewer data. (For one-layer compounds, the occurrence of different structural distortions, such as the coordinated tilting of $\mathrm{CuO}_{6}$ octahedra in $\mathrm{La}_{2} \mathrm{CuO}_{4}$, make such a comparison difficult.) For the two-layer compounds with insulating charge reservoir layers $\left(\mathrm{La}_{2} \mathrm{CaCu}_{2} \mathrm{O}_{6}, \mathrm{Tl}-1212, \mathrm{Bi}-2212, \mathrm{Tl}-2212\right.$, and $\left.\mathrm{Hg}-1212\right)$, the correspondence between $\mathrm{T}_{\mathrm{c}}$ and $\mathrm{CuO}_{2}$ plane buckling is remarkable. Flat planes lead to higher $T_{c}$ 's. A few degrees of buckling lowers $T_{c}$ substantially. The $\mathrm{HgBa}_{2} \mathrm{CaCu}_{2} \mathrm{O}_{6+\mathrm{x}}$ compound has a buckling angle of $179.4^{\circ}$, implying that a small increase in $T_{c}$ (perhaps $10 \mathrm{~K}$ ) would be achieved if the plane could be made perfectly flat.

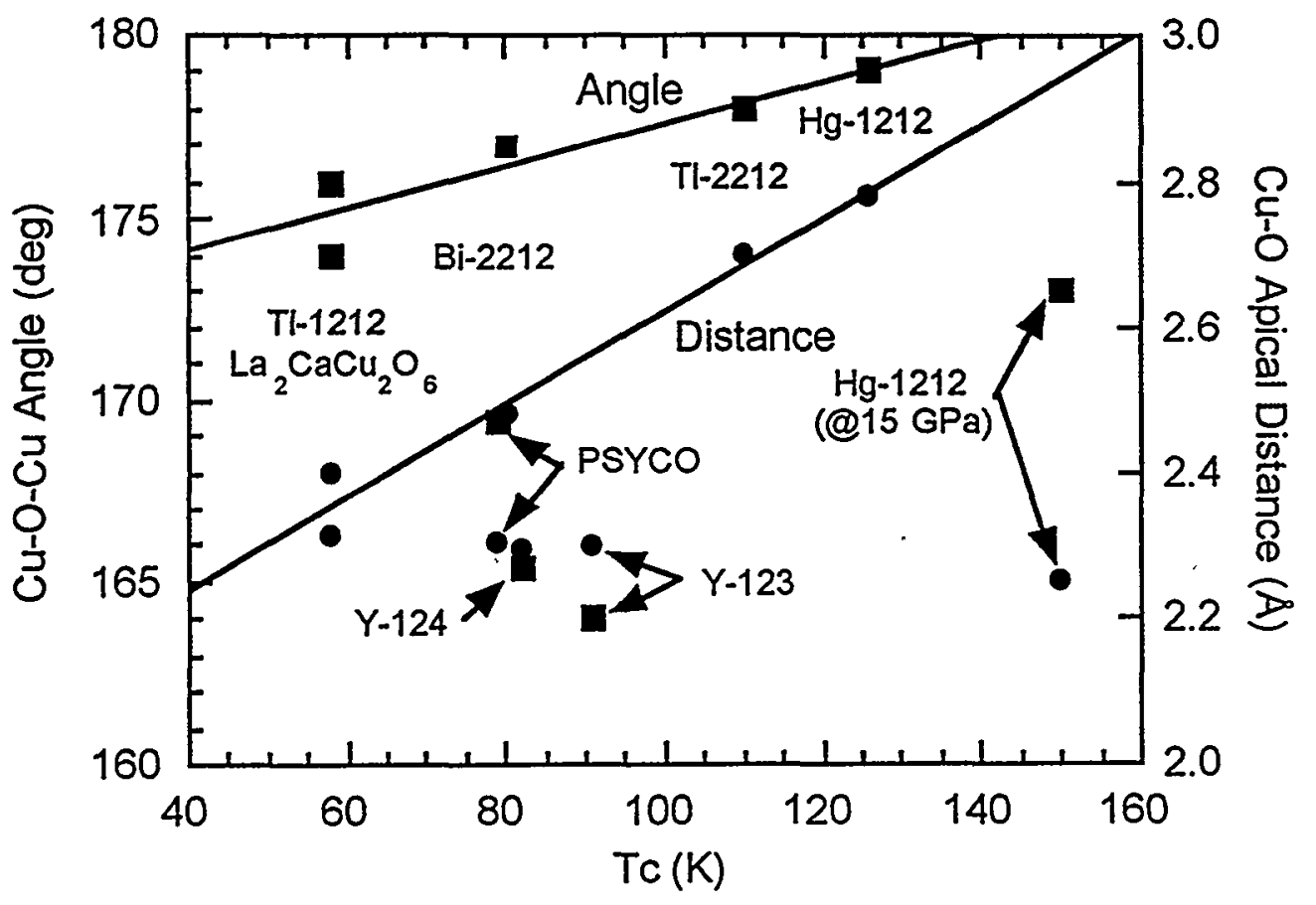

Fig. 1. Relationship between $\mathrm{T}_{\mathrm{c}}$, the $\mathrm{Cu}-\mathrm{O}-\mathrm{Cu}$ bucking angle of the $\mathrm{CuO}_{2}$ planes (square symbols), and the $\mathrm{Cu}-\mathrm{O}$ apical bond distance (round symbols) for compounds with two $\mathrm{CuO}_{2}$ layers.

A number of studies where the buckling angle has been varied while holding the doping level constant have confirmed this relationship between $T_{c}$ and buckling angle. The simplest and most direct confirmation comes from the studies of $\mathrm{La}_{2-\mathrm{x}} \mathrm{Sr}_{\mathrm{x}} \mathrm{CuO}_{4}$ vs. pressure by Yamada and Ido.[12] Pressure reduces the buckling angle of the $\mathrm{CuO}_{2}$ planes in the orthorhombic structure until a continuous transformation to a tetragonal structure with flat planes occurs. $T_{c}$ increases linearly with pressure until the planes become flat and then remains constant with pressure. Experiments by Dabrowski et al., where the buckling angle is varied through chemical substitution in $\mathrm{La}_{2-\mathrm{x}} \mathrm{M}_{\mathrm{x}} \mathrm{CuO}_{4}(\mathrm{M}=\mathrm{Nd}, \mathrm{Ca}, \mathrm{Sr})$ lead to the same conclusion.[13] The same behavior is observed in other high- $\mathrm{T}_{\mathrm{c}}$ compounds, such as the 123 materials, and there are several electronic structure calculations that provide an explanation in terms of how buckling affects the electronic density of states at the Fermi energy. This topic is reviewed in Ref. 14.

A recent report of the effect of structural disorder on $T_{c}$ may also be explained in terms of the effects of disorder on local buckling angle. Attfield et al. [15] showed that $T_{c}$ 
decreases systematically with increasing variance of the size of the charge reservoir cation in $\mathrm{Ln}_{1.85} \mathrm{M}_{0.15} \mathrm{CuO}_{4}$, where the combination of $\mathrm{Ln}(\mathrm{La}$ or $\mathrm{Nd})$ and $\mathrm{M}$ ( $\mathrm{Sr}, \mathrm{Ba}$, or $\mathrm{Ca}$ ) is chosen to conserve the doping level while introducing structural disorder because of the different sizes of the cations. They offered no fundamental explanation for why the resulting lattice strains lower $T_{c}$. I suggest that the suppression of $T_{c}$ is caused by increases in the buckling angle (which was not measured) in the local structure resulting from the strain.

Fig. 1 also illustrates the behavior of several two-layer compounds that violate the expected relationship between $T_{c}$ and buckling angle. These are all compounds where the charge reservoir layer is metallic. In this context, the concept of a metallic charge reservoir layer means that there are bands associated with this region of the structure that lie near the Fermi energy and, thus, contribute to the electronic properties. This is true for $\mathrm{YBa}_{2} \mathrm{Cu}_{3} \mathrm{O}_{6+\mathrm{x}}(\mathrm{Y}-123)$ if the $\mathrm{CuO}_{\mathrm{x}}$ chains are fully populated with oxygen $(\mathrm{x} \approx 1)$ and for $\mathrm{YBa}_{2} \mathrm{Cu}_{4} \mathrm{O}_{8}(\mathrm{Y}-124)$ and $\mathrm{Pb}_{2} \mathrm{Sr}_{2} \mathrm{Y}_{1-x} \mathrm{Ca}_{x} \mathrm{Cu}_{3} \mathrm{O}_{8+\delta}$ (PSYCCO), all of which have $\mathrm{Cu}$ in the charge reservoir layer. This observation suggests that metallization of the charge reservoir layer, which reduces the anisotropy in the normal-state conductivity and increases the c-axis coupling between $\mathrm{CuO}_{2}$ layers, can increase $\mathrm{T}_{\mathrm{c}}$ significantly. The most striking illustration of this phenomena is the behavior of the $\mathrm{HgBa}_{2} \mathrm{Ca}_{n}$ ${ }_{1} \mathrm{Cu}_{n} \mathrm{O}_{2 n+2+x}$ compounds at high pressure. For most high- $\mathrm{T}_{\mathrm{c}}$ compounds, pressure is thought to increase $T_{c}$ by promoting charge transfer; and, therefore; the effects of pressure are small for the composition that is optimally doped.[16] In contrast, pressure substantially raises the $T_{c}$ 's of the optimally-doped $n=1,2$, and $3 \mathrm{HgBa}_{2} \mathrm{Ca}_{n}$. ${ }_{1} \mathrm{Cu}_{n} \mathrm{O}_{2 \mathrm{n}+2+\mathrm{x}}$ compounds (by $30 \mathrm{~K}$ or more in each case). [17] This has been explained by band structure calculations based on the pressure-induced changes in the structures.[18] Pressure moves a band associated with the $\mathrm{HgO}_{\mathrm{x}}$ layer to the Fermi energy; i.e., pressure metallizes the charge reservoir layer and this contributes to an increased $\mathrm{T}_{c}$ in spite of the $\mathrm{CuO}_{2}$ plane buckling that results from the structural compression. The similarity to the other compounds with metallic charge reservoir layers is striking. As shown in Fig. 1, when pressure is applied to the Hg-1212 compound, which originally has an insulating blocking layer and obeys the expected behavior for those compounds, it transforms to a compound with a metallic blocking layer and has a higher $T_{c}$ in spite of the increased plane buckling. The ideal compound with flat $\mathrm{CuO}_{2}$ planes and a metallic charge reservoir layer has not yet been discovered.

\section{ELECTRONIC BARRIER TO ACHIEVING HIGHER $\mathrm{T}_{c}$}

These observations for the $\mathrm{HgBa}_{2} \mathrm{Ca}_{n-1} \mathrm{Cu}_{n} \mathrm{O}_{2 n+2+x}$ compounds provide critical insight into the challenge that must be met to increase the (ambient pressure) $T_{c}$ of layered copper-oxide compounds beyond the present record of $135 \mathrm{~K}$. One must achieve a structure with flat $\mathrm{CuO}_{2}$ planes; the chemistry of the charge reservoir layer must be adjusted to achieve optimal doping of the $\mathrm{CuO}_{2}$ planes; and the charge reservoir layer must also be metallic. In terms of the electronic structure, I assume that the latter two criteria mean that bands associated with the $\mathrm{CuO}_{2}$ planes and the charge reservoir layer must simultaneously be at the Fermi energy. These criteria cannot, a priori, simultaneously be achieved by adjusting a single chemical variable. Hence, it is clear why both chemical doping and pressure must be used to achieve the highest $T_{c}$ in the $\mathrm{HgBa}_{2} \mathrm{Ca}_{n-1} \mathrm{Cu}_{n} \mathrm{O}_{2 n+2+x}$ compounds -- two variables are needed to adjust two bands to lie at the Fermi energy.

Recent work on the $\mathrm{HgBa}_{2} \mathrm{CuO}_{4+x}$ compound suggests that electronically-driven structural distortions may increase the difficulty of making the ideal high- $\mathrm{T}_{\mathrm{c}}$ material. Fig. 2 
shows the $\mathrm{T}_{c}$ and unit cell volume of $\mathrm{HgBa}_{2} \mathrm{CuO}_{4+x}$ as a function of the internal structural parameter $[z(\mathrm{Ba})-\mathrm{z}(\mathrm{O} 2)]$.[19] This structural parameter, which generally decreases with increasing oxygen content, is a measure of the charge transfer as oxygen is added to the compound. At the maximum $T_{c}$, as the material passes from the under-doped to the over-doped state, there is a region where $T_{c}$ remains constant and the unit cell volume increases while oxygen is added. This anomalous increase in the unit cell volume changes the structure so that the band associated with the Hg-containing charge reservoir layer does not lie at the Fermi energy; i.e., the structure distorts, by increasing its cell volume, to avoid placing this band at the Fermi energy. The application of pressure, which reduces the cell volume, can be viewed as removing this electronically-driven structural distortion and moving the charge-reservoir band to the Fermi energy.

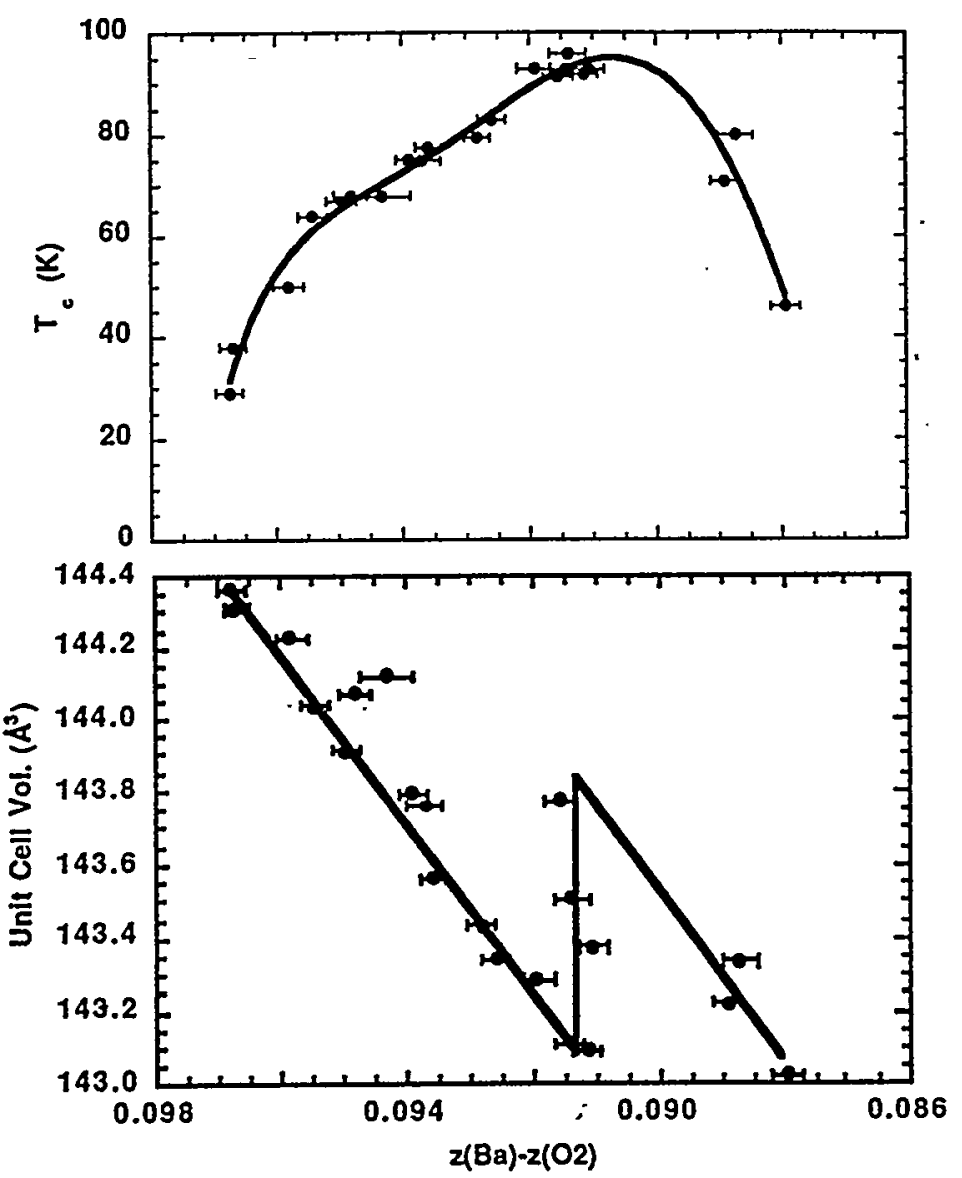

Fig. 2. $\mathrm{T}_{\mathrm{c}}$ (top frame) and unit cell volume (bottom frame) of $\mathrm{HgBa}_{2} \mathrm{CuO}_{4+x}$ vs. the structural parameter $[z(\mathrm{Ba})-\mathrm{z}(\mathrm{O} 2)]$, which is a measure of the charge transfer (see text).

Such behavior (structural distortion that lowers the density of states at the Fermi energy) is common in conventional superconductors, but has seldom been explicitly studied in the high- $\mathrm{T}_{c}$ materials. However, this behavior is not unique to the $\mathrm{HgBa}_{2} \mathrm{CuO}_{4+\mathrm{x}}$ compound. A recent study of a chemically-substituted 123 compound in which both the under-doped and over-doped states can be accessed by changing the oxygen content 
shows a similar electronically-driven structural distortion at the maximum $\mathrm{T}_{\mathrm{c}}$.[14] In the 123 compound, the structural distortion manifests itself as an increased buckling of the

$\mathrm{CuO}_{2}$ planes, which we have already argued will lower $\mathrm{T}_{\mathrm{c}}$.

The challenge of achieving a higher $\mathrm{T}_{\mathrm{c}}$ in the layered copper-oxide compounds is, thus, not an easy task. The desired criteria are well understood, but more than one chemical and/or structural variable is needed to simultaneously achieve the ideal structural and electronic properties. Recent work suggests that, as this ideal compound is approached, electronically-driven structural distortions can suppress $\mathrm{T}_{c}$ by distorting the structure to avoid the ideal structural and/or electronic properties. In spite of these challenges, the $T_{\mathfrak{c}}$ of over $160 \mathrm{~K}$ achieved in the $\mathrm{HgBa}_{2} \mathrm{Ca}_{2} \mathrm{Cu}_{3} \mathrm{O}_{8+\mathrm{x}}$ compound at high pressure (in a compound with buckled planes) argues that further increases in $\mathrm{T}_{\mathrm{c}}$ at ambient pressure should be possible.

Acknowledgments: The work reviewed here was supported by the U. S. Department of Energy, Energy Research - Materials Sciences, contract No. W-31-109-ENG-38 and the National Science Foundation, Office of Science and Technology Centers, grant No. DMR 91-20000. I wish to especially thank D. G. Hinks, O. Chmaissem, P. G. Radaelli, J. L. Wagner, H . Shaked, and B. Dabrowski, who have been key collaborators in much of the work.

\section{REFERENCES}

1. R. J. Cava, Physica C 282-287, 27 (1997).

2. A. Schilling, M. Cantoni, J. D. Guo, \& H. R. Ott, Nature 363, 56 (1993).

3. R. J. Cava et al., Physica C 165, 419 (1990).

4. J. D. Jorgensen et al., Phys. Rev. B 38, 11337 (1988).

5. J. D. Jorgensen et al., Physica C 167, 571 (1990).

6. Y. Maeno et al., Nature 328, 512 (1987); J. M. Tarascon et al., Phys. Rev. B 37, 7458 (1988); R. S. Howland et al., Phys. Rev. B 39, 9017 (1989).

7. H. Shaked et al., Phys. Rev. B 48, 12941 (1993) and the references cited therein.

8. J. L. Wagner et al., Physica C 210, 447 (1993).

9. P. G. Radaelli et al., Physica C 216, 29 (1993).

10. J. L. Wagner et al., Phys. Rev. B 51, 15407 (1995).

11. J. D. Jorgensen et al., in Recent Developments in High Temperature Superconductivity, edited by J. Klamut et al., Lecture Notes in Physics, Vol. 475 (Springer-Verlag, 1996) p. 1.

12. Y. Yamada and M. Ido, Physica C 203, 240 (1992).

13. B. Dabrowski et àl., Phys. Rev. Lett. 76, 1348 (1996).

14. O. Chmaissem et al., Nature 397, 45 (1999).

15. J. P. Attfield, A. L Kharlanov, \& J. A. McAllister, Nature 394, 157 (1998).

16. J. S. Schilling and S. Klotz, in Physical Properties of High Temperature Superconductors, Vol. III, edited by D. M. Ginsberg (World Scientific Publ., Singapore, 1992) p. 59.

17. L. Gao et al., Phys. Rev. B 50, 4260 (1994).

18. D. L. Novikov, O. N. Myrasov, \& A. J. Freeman, Physica C 222, 38 (1994); D. J. Singh \& W. E. Pickett, Physica C 233, 237 (1994).

19. J. D. Jorgensen et al., in High Temperature Superconductors and Novel Materials, edited by G. Van Tendeloo et al. (NATO Book Series, Kluwer Academic Publ., B. V., 1999) p. 109. 